International Journal of Engineering \& Technology, $7(2.22)(2018) 27-30$
International Journal of Engineering \& Technology
Website: $w$ ww.sciencepubco.com/index.php/IJET
Research paper

\title{
A multivariate Gaussian modeling for counting bananas
}

\author{
Senthilarasi $\mathbf{M}^{1^{*}}$, Navarasy $\mathbf{T} \mathbf{M}^{2}$, RamKumar $\mathrm{C}^{3}$, Mohamed Mansoor Roomi $\mathrm{S}^{4}$ \\ ${ }^{1}$ Research scholar, Thiagarajar college of Engineering, Madurai \\ ${ }^{2} U G$ Student, Thiagarajar college of Engineering, Madurai \\ ${ }^{3} U G$ Student, Thiagarajar college of Engineering, Madurai \\ ${ }^{4}$ Assistant professor, Thiagarajar college of Engineering, Madurai \\ *Corresponding author E-mail: arasiece@gmail.com
}

\begin{abstract}
Agriculture industries play an important role in economic development. Many post harvest techniques are performed in these industries. Especially in fruit industries, grading is an important process. As per the grading standard, banana hands must have minimum of twelve banana fingers. In the current scenario, the banana fingers are counted manually which is laborious. In order to reduce manual work, this paper proposes an algorithm for performing an automatic counting system of banana fingers in a bunch/hand using image processing algorithms. The parameters of the Gaussian model is acquired from the samples of fruit distal ends. A multivariate Gaussian modeling is accomplished on HSV color model to detect the distal end of banana fingers using maximum likelihood detection.
\end{abstract}

Keywords: Gaussian modeling ; HSV color space; Maximum likelihood detection ; Multivariational approach.

\section{Introduction}

Nowadays, information and communication technology is developing all over the world. Each and every field is working with the dependency on multiple disciplines like image processing is aiding in agro, automobile industries and etc.. Banana is the most important product of the Asia and Pacific area. Banana is one of the most important fruit crops grown in India. With the highest annual production of 265 lakh tones, banana is the most cultivated fruit of India. It is grown all over the states and is available all throughout the year. Main banana producing states are Tamil Nadu, Maharashtra, Karnataka, Gujarat, Andhra Pradesh, Bihar, Assam and Madhya Pradesh. The main varieties of banana involve Dwarf Cavendish; Robusta; Rasthali; Poovan; Nendran; Red Banana; Karpurvalli; Pachanadan, Virupakshi etc. Banana ranks second in cultivation area and first in production amount. Mudhalwadkar et al. have discussed the data driven modeling for banana ripeness assessment. In this paper, bananas are classified based on dielectric spectroscopy and image processing techniques. Those result is achieved with fuzzy logic controller. This process is nondestructive, quick, reliable and effective[1]. Rajendra Hegadi et al. has proposed an easy monitoring system to monitor the color indices of the bananas and to identify ripening stage of banana; this information will be sent to the monitoring person automatically to the remote area. The paper has presented an effective system for wireless monitoring of the processing of the banana fruits in the store room [2]. Senthilarasi et al. has detected the Musa Species in Fruit Industry using shape based approach. In this paper, they have done a research work on image processing algorithm that ensures automatic discrimination of banana (Musa Species.) from other fruits like Citrus, Apple, and Pomegranate. The accuracy rate is 95\% [3]. Nur Badariah Ahmad Mustafa has discussed the Image Processing of an Agriculture Produce: Determination of Size and Ripeness of a Banana. In this paper the key idea is the edge detection and the color changes that help us in determining the quality of the banana[4]. Anisha Syal has detect the apple fruit and counting using Computer Vision Techniques. In this paper, they have counted the fruits using minimum Euclidean distance based segmentation technique for segmenting the fruit region from the input image[5]. Chi Cuong Tran et al. have designed the automatic counting dragon fruit using adaptive thresholds for image segmentation and shape analysis. In this paper, the problem of counting the correct number of fruits per tree to accurately estimate the yield of dragon fruit in the farm is solved[6]. In the above analysis of research papers, it is understood that the image processing plays an important role in fruit industries. The literatures have discussed the banana ripeness ,banana discrimination, and counting fruits on the tree for other fruits like apple, dragon fruit, citrus and etc. The grading of banana fruit requires a personnel to confirm that the number of banana fingers in a hand is greater than 12 numbers. Counting in banana fruit industries are carried out by human evaluation which may not be effective with respect to time, correctness due to the laborious nature. Hence an automated system for counting is necessitated to count the number of banana fingers with the help of their distal ends because banana fruit is self occluded object which cannot be counted by seeing that on either top or bottom. The tip/distal ends of the banana are taken as a cue for counting in the proposed paper. The main contributions are

- Collection of banana distal end patches for modeling

- Model the banana fruit tips as trivariate Gaussian function.

- Detection of banana fruit distal ends by applying Maximum likelihood.

The organization of paper is as follows, section II explains about the materials and methods, section III explains about the results and discussions, section IV explains about the conclusion.

\section{Materials and Methods}


In the industry, the fruits are passed by the conveyor from one unit to the other. A camera installed at the chamber captures the distal ends of banana while their transit. These images are fed to the processor for further image analysis to inspect the count of banana fingers in a hand for grading. If a banana hand has less than 12 banana fingers then those bunches are to be separated.

\subsection{Samples}

Three Indian species of banana (Musa) such as Nendran, Rasthali and Nadu are considered here. Some of the images are collected from the internet and the rest are captured at the laboratory. The scope of the proposed work is limited to uniform background and illumination.

\subsection{Image Acquisition}

In the prototype of the proposed work, the RGB images of fruits are captured. The images are acquired at the controlled environment to limit the effects of illumination change. As a standard capture condition, images were taken on a particular place with the background of white coloured non-reflective cloth. Each image of the fruit is captured in different positions and views using NikonD3100 camera.

\subsection{Process flow}

The objective of the proposed work is to count the number of bananas in a bunch using multivariate approach. For that, first the tip of each banana is to be segmented as ROI from non fruit end regions. The flow of the proposed method is shown in figure 1 .

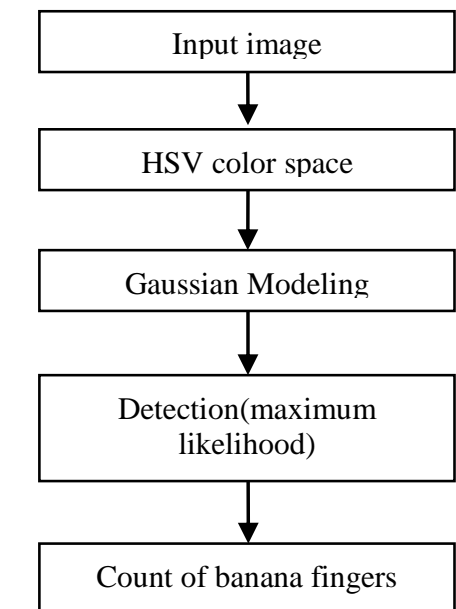

Fig.1: Flow Chart of Image Processing System

\subsubsection{HSV colour space conversion}

The input RGB (red, green, blue) images are converted into HSV (hue, saturation, value) colour space. Hue represents the colour shade of an image. Saturation represents the gray colour of an image from 0 percent to 100 percent. Value represents the brightness or intensity value of an image. Both the fruit end samples and non fruit end samples is converted into hsv colour space for modeling.

\subsubsection{Gaussian modeling}

For a Gaussian modeling, the parameters are mean and variance. It is evident that when a large amount of data are collected then it follows a Gaussian distribution. In this work, we have collected many fruit end and non-fruit end patches of size $25 \times 25$ to model it as a Gaussian function. A Gaussian function can be either univariate or multivariate Gaussian modeling. If the object depends on the single parameter hence it is known as univariate Gaussian modeling. If the object depends on more than two parameters hence it is known as trivariate or multivariate Gaussian modeling. In this scenario, the tips of the banana fruit end are colour modelled in HSV colour space. Hence, this approach is a trivariate Gaussian model. Here the variables are hue, saturation \& value. The multivariate Gaussian function is expressed as

$$
P(X ; \mu, \Sigma)=\frac{1}{(2 \Pi)^{\frac{n}{2}}|\Sigma|^{\frac{1}{2}}} e\left(-\frac{1}{2}(X-\mu)^{T} \Sigma^{-1(X-\mu)}\right)
$$

where Mean $\mu$, Covariance matrix $\Sigma, \mathrm{x}$ is a random variable of $\mathrm{x}=[\mathrm{x} 1, \mathrm{x} 2, \ldots \ldots . \mathrm{xn}]^{\mathrm{T}}$

\subsubsection{Maximum Likelihood Detection}

The detection of fruit end from non fruit end region is implemented using maximum likelihood. In maximum likelihood, the extracted model parameters of fruit end and non-fruit end are use. The likelihood of HSV values of query image belonging to fruit end and non-fruit end is estimated with modelled parameters of fruit end and non-fruit end. A decision is made against fruit end detection if the likelihood of fruit end is higher than the likelihood of non-fruit end. This decision leads to the segmentation of banana fruit distal ends.

\subsubsection{Count of banana fingers}

The segmented banana fruit ends are the region of interest(ROI). Morphological operations such as erode is used to prune the segmented result for clearing overlaps. Then by the connected component analysis, the number of blobs are counted. This gives the number of banana fingers in a banana hand.

\subsection{Performance measure}

Seven different performance measures such as PSNR(peak signal to noise ratio), MAD(Mean absolute difference), MSE(mean square error), TP(true positive), TN(true negative), $\mathrm{FP}$ (false positive) and FN(false negative) are considered here. The formulae are:

$$
\begin{aligned}
& M S E=\frac{1}{m^{*} n} \sum_{i=1}^{m} \sum_{j=1}^{n}[I(i, j)-I(i, j)]^{2} \\
& P S N R=20 \log _{10}[255 / M S E] \\
& M A D=\frac{\sum_{i=1}^{m} \sum_{j=1}^{n}\left|y_{i}-y_{j}\right|}{n(n-1)} \\
& \mathrm{TP}=\operatorname{sum}(\text { prediction}==\text { True AND groundtruth }==\text { True) } \\
& \mathrm{FP}=\operatorname{sum}(\text { prediction}==\text { True AND groundtruth }==\text { False }) \\
& \mathrm{TN}=\operatorname{sum}(\text { prediction}==\text { False AND groundtruth }==\text { False }) \\
& \mathrm{FN}=\operatorname{sum}(\text { prediction}==\text { False AND groundtruth==True) }
\end{aligned}
$$

\section{Results and Discussions}

The banana database comprises of Nendran, Rasthali and Nadu. Sample images of banana are shown in Figure2. From the banana database, the patches of the banana fruit end is cropped for modeling. Also, few non-fruit end patches are collected from other part of banana. The patch size is taken as $25 \times 25$. Here, the number of patches for fruit end is 2322 images. Therefore, it comprises of 2322 x 25 x 25 (14,51,250 data samples) for modeling. Similarly, the number of modeling patches for non fruit end is 2184 images(13,65,000 data samples). The RGB images of patches of fruit end and non fruit end are shown in Figure 3 and 4 respectively. Few patches of fruit end and non-fruit end in HSV colour space is 
shown in Figure 5 and 6 respectively. The mean and covariance matrix for both fruit end and non fruit end are given in the Table1 and 2 respectively.

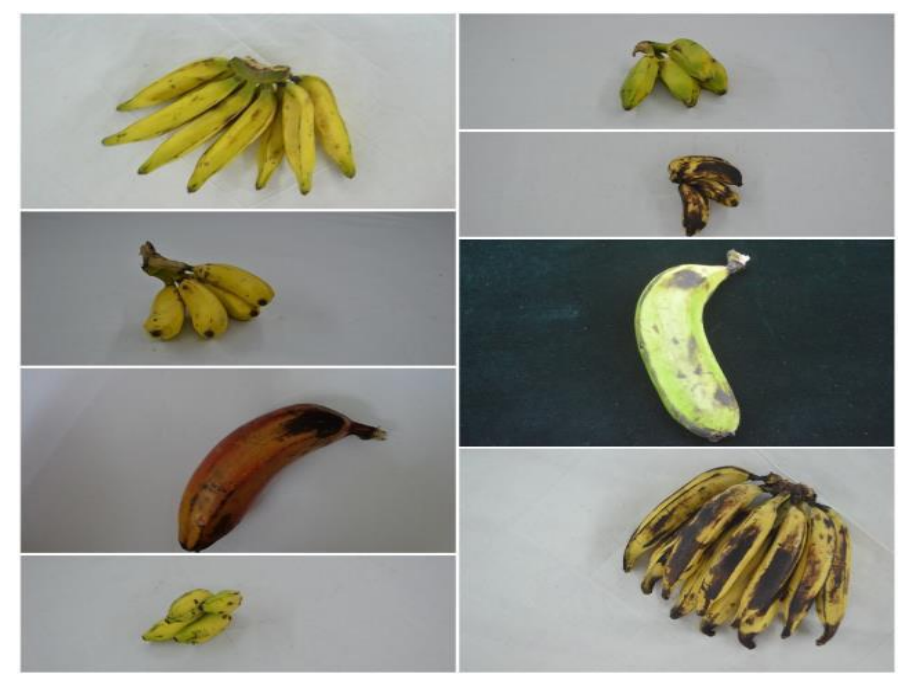

Fig.2: Training Set

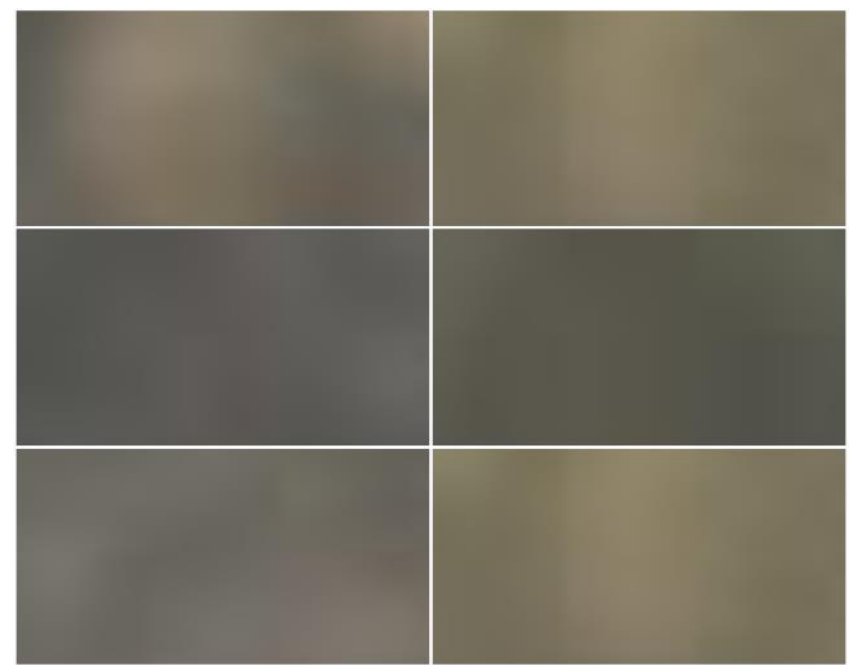

Fig.3: Samples for Fruit End

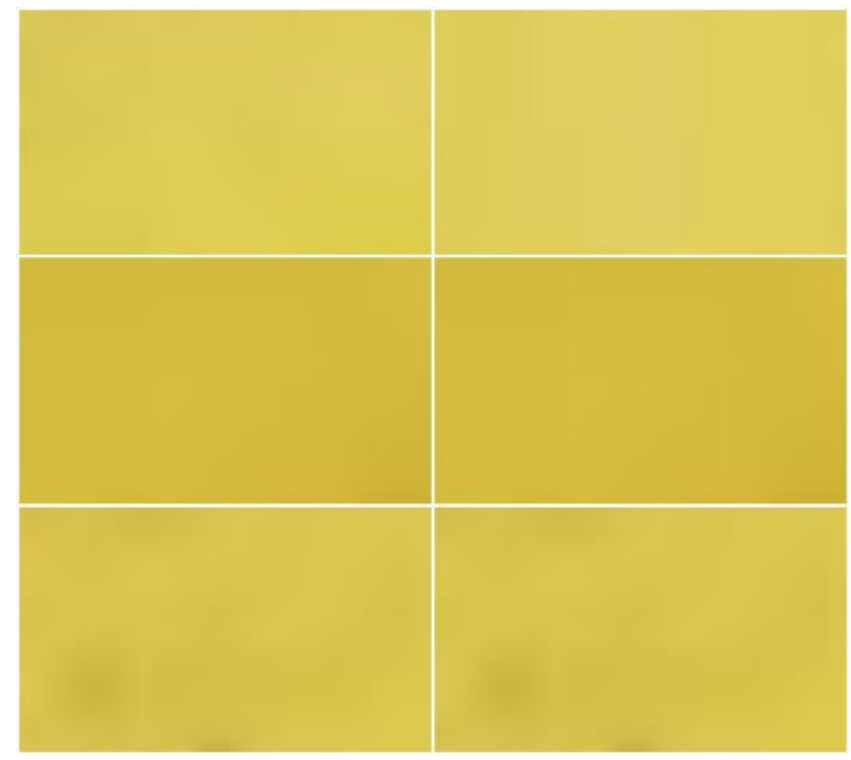

Fig.4: Samples for Non Fruit End
Table 1: Parameters of Fruit End

\begin{tabular}{|l|l|l|l|}
\hline & HUE & SATURATION & VALUE \\
\hline MEAN & 0.2499 & 0.2760 & 0.3545 \\
\hline COVARIANCE & {$\left[\begin{array}{llll}0.0629 & -0.0220 & 0.0012 & \\
\text { MATRIX } & -0.0220 & 0.0577 & -0.0170 \\
& 0.0012 & -0.0170 & 0.0337\end{array}\right]$} \\
\hline
\end{tabular}

Table 2: Parameters of Non Fruit End

\begin{tabular}{|c|c|c|c|}
\hline & HUE & SATURATION & VALUE \\
\hline MEAN & 0.1603 & 0.7233 & 0.6170 \\
\hline COVARIANCE & \multicolumn{3}{|c|}{$\left[\begin{array}{lll}0.0131 & -0.0138 & -0.0046\end{array}\right.$} \\
\hline MATRIX & \multicolumn{3}{|c|}{$\begin{array}{ccc}-0.0138 & 0.0601 & -0.0101 \\
-0.0046 & -0.0101 & 0.03731\end{array}$} \\
\hline
\end{tabular}
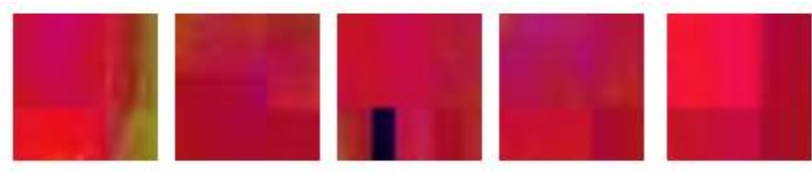

Fig.5: Samples of Fruit End patches in HSV
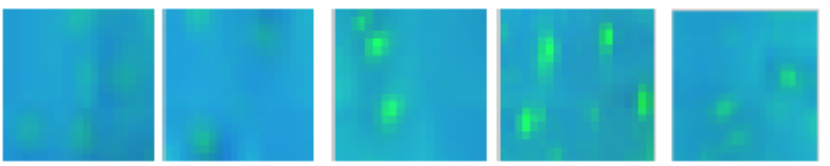

Fig.6: Samples of Non-Fruit End patches in HSV

The test image is shown in Figure 7.The test image is converted to HSV colour space as shown in Figure 8. The detection of distal end using multivariate Gaussian modeling and maximum likelihood detection is depicted in Figure 9. Finally, the number of bananas is counted using the extracted tip portion as shown in Figure 10.

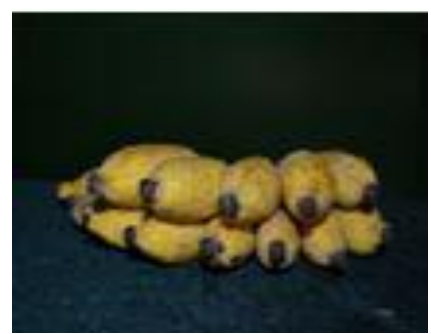

Fig.7: Test Image

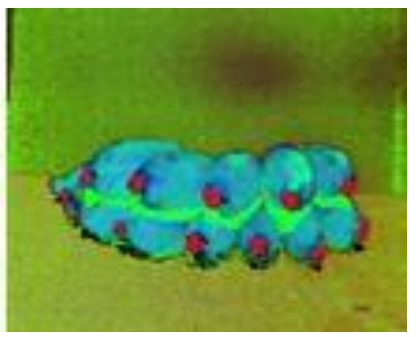

Fig.8: Test Image HSV 


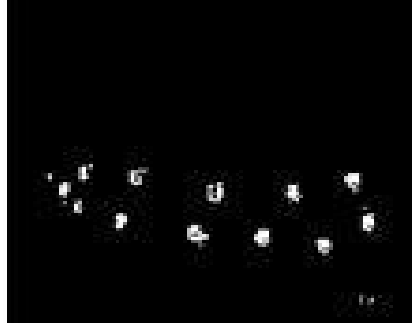

Fig.9: Extracted Image

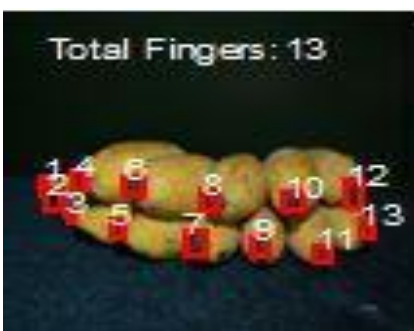

Fig.10: Output Image

Table 3: Performance measures

\begin{tabular}{|l|l|l|l|l|l|l|l|l|l|}
\hline $\begin{array}{c}\text { Sam } \\
\text { ple } \\
\text { im- } \\
\text { ages }\end{array}$ & $\begin{array}{c}\text { PSN } \\
\text { R }\end{array}$ & $\begin{array}{c}\text { MA } \\
\text { D }\end{array}$ & $\begin{array}{c}\text { MS } \\
\text { E }\end{array}$ & TP & TN & FP & FN & $\begin{array}{c}\text { Accu- } \\
\text { racy }\end{array}$ & $\begin{array}{c}\text { Er- } \\
\text { ror }\end{array}$ \\
\hline 1 & 118. & 0.02 & 0.02 & 0.0 & 0.9 & 0.0 & 0 & 0.972 & 0.0 \\
& 03 & 66 & 66 & 22 & 50 & 26 & & & 26 \\
\hline 2 & 116. & 0.03 & 0.03 & 0.0 & 0.9 & 0.0 & 0.0 & 0.961 & 0.0 \\
& 43 & 85 & 85 & 14 & 47 & 30 & 08 & & 38 \\
\hline 3 & 115. & 0.04 & 0.04 & 0.0 & 0.9 & 0.0 & 0.0 & 0.95 & 0.0 \\
& 41 & 86 & 86 & 16 & 34 & 45 & 02 & & 47 \\
\hline 4 & 114 & 0.06 & 0.06 & 0.0 & 0.9 & 0.0 & 0 & 0.937 & 0.0 \\
& 4.3 & 19 & 19 & 16 & 21 & 61 & & & 61 \\
\hline 5 & 116. & 0.03 & 0.03 & 0.0 & 0.9 & 0.0 & 0.0 & 0.961 & 0.0 \\
& 50 & 78 & 78 & 14 & 47 & 31 & 06 & & 37 \\
\hline 6 & 116. & 0.03 & 0.03 & 0.0 & 0.9 & 0.0 & 0 & 0.965 & 0.0 \\
& 99 & 38 & 38 & 12 & 53 & 33 & & & 33 \\
\hline
\end{tabular}

In the above table, PSNR value ranges from 114 to 118, MAD value is 2.6 to $6.1 \%$, MSE value is range from 2.6 to $6.1 \%$,Accuracy is $95.7 \%$ and Error is $4 \%$,According to those values, MAD and MSE values are more or less similar.

\section{Conclusion}

This paper proposes an automatic system for counting banana fingers of a hand which could help the fruit processing industries to adhere to the grading standard of banana fruit. Also, this could help the seller to price it during billing. The proposed method counts the number of bananas in a bunch. The input image is converted to HSV colour space. The resultant HSV colour image is modelled by multivariate Gaussian mapping and the results are compared by maximum likelihood detection. The connected component analysis on the detected region of interest gives the count of banana fingers in a hand.

\section{References}

[1] Maithilee Nagesh Kulkarni, Dr. (Mrs.) R. P. Mudhalwadkar,” Data driven modeling for banana ripeness Assessment", International Conference on Intelligent Computing and Control Systems,2017.

[2] Amit Verma, Rajendra Hegadi, Kamini Sahu," Development of an Effective System for Remote Monitoring of Banana Ripening Process",IEEE International WIE Conference on Electrical and Computer Engineering(WIECON-ECE),2015.

[3] M.Senthilarasi, S.Md.Mansoor Roomi and M.R.H.Prasanna," Shape based approach for Detecting Musa Species in Fruit In- dustry", Sixth International Conference on Advanced Computing(1CoAC), 2014.

[4] Nbadariah, NAShikin, syedkhaleel, AidilAzwin, Zaipatimah, Zainul," Image Processing of an Agriculture Produce: Determination of Size and Ripeness of a Banana", IEEE,2008.

[5] Anisha Syal, Divya Garg, Shanu Sharma," Apple Fruit Detection and Counting Using Computer Vision Techniques", IEEE International Conference on Computational Intelligence and Computing Research,2014

[6] Chi Cuong Tran, Dinh Tu Nguyen, Hoang Dang Le, Quoc Bao Truong, Quoc Dinh Truong," Automatic dragon fruit counting using adaptive thresholds for image segmentation and shape analysis", 4th NAFOSTED Conference on Information and Computer Science, 2017.

[7] Chuong B. Do," The Multivariate Gaussian Distribution", October 10, 2008.

[8] Veena Hallur, BhagyashreeAtharga, Amruta Hosur, Bhagyashree Binjawadagi, K. Bhat," Design and development of a portable instrument for the detection of artificial ripening of banana fruit, Proceedings of International Conference on Circuits, Communication, Control and Computing (I4C 2014). [9]Monika Jhuria, Ashwani Kumar, Rushikesh Borse," Image Processing for Smart Farming:Detection of Disease and Fruit Grading",IEEE Second international Conference on Image Information Processing"(ICIIP),2013. 